\author{
Review Article
}

\title{
Prophylaxis and treatment of hepatitis B in immunocompromised patients
}

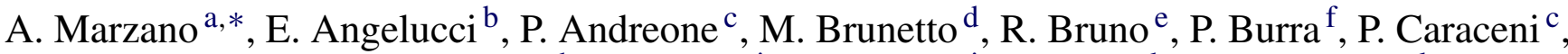 \\ B. Daniele ${ }^{\text {g, V. Di Marco }}{ }^{\text {h }}$, F. Fabrizi ${ }^{i}$, S. Fagiuoli ${ }^{j}$, P. Grossi $^{\text {k }}$, P. Lampertico ${ }^{\text {, }}$,

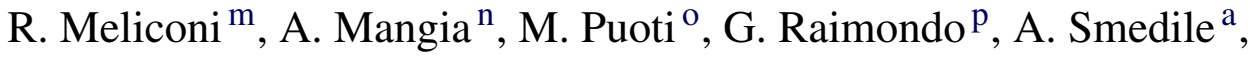 \\ for the Italian Association for the Study of the Liver (A.I.S.F.) \\ a Division of Gastroenterology and Hepatology, AO San Giovanni Battista, Torino, Italy \\ b Hematology Division and Haemopoietic Stem Cell Transplant Centre, "Armando Businco" Cancer Centre, Cagliari, Italy \\ ${ }^{\mathrm{c}}$ Department of Internal Medicine, Cardio-Angiology and Hepatology, University of Bologna, Italy \\ ${ }^{\mathrm{d}}$ Division of Gastroenterology and Hepatology, AOU Pisana, Pisa, Italy \\ e Institute of Infectious and Tropical Diseases, University of Pavia, Italy \\ ${ }^{\mathrm{f}}$ Department of Surgical and Gastroenterological Science, University of Padova, Italy \\ g Medical Oncology Unit, Ospedale G. Rummo, Benevento, Italy \\ ${ }^{\mathrm{h}}$ Division of Gastroenterology and Hepatology, University of Palermo, Italy \\ i Division of Nephrology and Dialysis, Maggiore Hospital, IRCCS, Milan, Italy \\ j Division of Gastroenterology, Ospedali Riuniti, Bergamo, Italy \\ ${ }^{\mathrm{k}}$ Division of Infectious and Tropical Diseases, Department of Clinical Medicine, University of Insubria, Varese, Italy \\ ${ }^{1}$ Division of Gastroenterology, IRCCS Maggiore Hospital, University of Milan, Italy \\ $\mathrm{m}$ Immunology and Genetics Laboratory, Istituti Ortopedici Rizzoli, Bologna, Italy \\ ${ }^{n}$ Division of Gastroenterology, Ospedale Casa Sollievo della Sofferenza, IRCCS, San Giovanni Rotondo, Italy \\ ${ }^{\circ}$ Institute of Infectious and Tropical Diseases, University of Brescia, Italy \\ $\mathrm{p}$ Department of Internal Medicine, University of Messina, Italy \\ Received 3 May 2006; accepted 18 December 2006 \\ Available online 23 March 2007
}

\begin{abstract}
The literature on hepatitis B virus (HBV) in immunocompromised patients is heterogeneous and referred mainly to the pre-antivirals era. Today a rational approach to the problem of hepatitis B in these patients provides for: (a) the evaluation of HBV markers and of liver condition in all subjects starting immunosuppressive therapies (baseline), (b) the treatment with antivirals (therapy) of active carriers, (c) the pre-emptive use of antivirals (prophylaxis) in inactive carriers, especially if they are undergoing immunosuppressive therapies judged to be at high risk, (d) the biochemical and hepatitis B surface antigen (HBsAg) monitoring (or universal prophylaxis, in case of high risk immunosuppression) in subjects with markers of previous contact with HBV (HBsAg negative and anti-HBc positive), in order to prevent reverse seroconversion.

Moreover it is suggested a strict adherence to criteria of allocation based on the virological characteristics of both recipients and donors in the general setting of transplants and in liver transplantation the universal prophylaxis with nucleos(t)ides analogues (frequently combined with specific anti-HBV immunoglobulins) in HBsAg positive candidates and in HBsAg negative recipients of anti-HBc positive grafts.
\end{abstract}

(C) 2006 Editrice Gastroenterologica Italiana S.r.l. Published by Elsevier Ltd. All rights reserved.

Keywords: Antivirals; HBV; Immunosuppression; Transplants

\footnotetext{
* Corresponding author at: SCDU of Gastroenterology and Hepatology, AO San Giovanni Battista, Corso Bramante 88, 10125 Torino, Italy. Tel.: +39011 6335561; fax +390116335927.

E-mail address: alfredomarzano@yahoo.it (A. Marzano).
} 


\section{Introduction}

Immunosuppression due to the underlying disease or to drugs used in autoimmune diseases, anticancer therapy and in organ transplants can influence the hepatitis B virus (HBV), both in terms of reactivation and in terms of the acceleration of a pre-existing chronic hepatitis.

In this situation, the possibility of HBV relapse has been known for years, with clinical manifestations ranging from self-limiting anicteric to fulminant forms or to chronic hepatitis with an accelerated clinical course towards liver decompensation. In most cases, hepatitis B develops at the time of immune reconstitution as a consequence of the antiviral immune response and less frequently at the time of the enhanced replication during massive immunosuppression. Moreover hepatitis reactivation may influence the continuation of the specific treatments and the survival of immunosuppressed or transplanted patients [1].

The risk of clinical events is mainly observed in overt carriers of HBV, but can also develop in the "occult" condition of infection which has been widely described in the literature of the last decade [2].

Progress in the diagnostic procedures of the various virological conditions associated with HBV and in particular the recent availability of effective antiviral treatments has brought this problem to the fore although it is still debated.

This encouraged the Italian Association for the Study of the Liver (AISF) to organize a Consensus conference according to the Italian Institute of Health guidelines (www.pnlg.it) (Table 1), which was held in Turin on May 13th and 14th, 2005. The indications reported below are the conclusions which emerged during and after the meeting, from the systematic review of the literature and from the multi-disciplinary debate.

\section{Definitions}

\subsection{Virological characteristics}

Persistant HBV infection is defined as overt when the hepatitis B surface antigen (HBsAg) is present in amounts well-detectable by sensitive immune assays and occult in HBsAg negative subjects with evidence of intrahepatic and/or serum HBV DNA [2]. In occult carriers, HBsAg can be completely absent or undetectable for very low amounts or polymorphisms.

\subsubsection{HBV carriers (HBsAg positive)}

In accordance with the international definitions, they can be identified as: (1) active carriers, in presence of hepatitis $\mathrm{B}$ envelope antigen ( $\mathrm{HBeAg}$ ) or of antibody to $\mathrm{HBeAg}$ (anti-HBe) and of a viral load $\geq 20,000 \mathrm{IU} / \mathrm{mL}$, according to the most recent standardisations; this condition is associated with the presence of hepatic disease in the most part of cases (AIII), or (2) inactive carriers, in case of subjects HBeAg negative and anti-HBe positive, whose alanine aminotransferase (ALT) levels are persistently within the normal range, HBV DNA below 20,000 IU/mL and antibody to $\mathrm{HBcAg}$ (anti$\mathrm{HBc}$ ) IgM levels $<0.20 \mathrm{IMx}$ index. In the majority of these subjects, the histological finding, when available, does not reveal a significant liver disease (necroinflammatory score $<4$ histology activity index (HAI)), while in a small minority of cases it is possible to observe the effects of a chronic liver disease which became silent spontaneously or following antiviral treatment $[3,4]$ (BIII).

\subsubsection{Occult $H B V$ carriers (HBsAg negative)}

The difficulty in determining HBV DNA in the liver biopsy (frequently not justified in subjects without clinical signs of hepatitis), the rare presence of detectable viremia in serum even with sensitive techniques, and the frequent presence in occult carriers of markers of previous contact with the HBV (anti-HBc \pm antibody to HBsAg (anti-HBs)), leads to consider all anti-HBc (anti-core) positive subjects as potential occult carriers. Instead there are no serum determinants in the minority (about 20\%) of occult carriers who are negative for all HBV markers.

\subsection{Virological events}

In HBV carriers (occult or overt), the following virological events are considered significant: (1) in anti-core subjects the re-emergence of HBsAg (seroreversion) (AIII), (2) in inactive carriers the appearance of a significant viremia ( $\geq 20,000 \mathrm{IU} / \mathrm{mL}$ ) (reactivation), as this is frequently associated with liver damage due to HBV (AIII), (3) in active carriers the persistence of a significant viremia $(\geq 20,000 \mathrm{IU} / \mathrm{mL}$ in $\mathrm{HBeAg}$ positive patients and $>2000 \mathrm{IU} / \mathrm{mL}$ in $\mathrm{HBeAg}$ negative subjects with chronic

Table 1

Levels of evidence and degree of substantiation (PNGL, www.pnlg.it)

\begin{tabular}{llll}
\hline Evidence levels from & \multicolumn{2}{l}{ Degree of substantiation: recommended procedure } \\
\hline I & RCTs and/or revision of RCTs & A & Strongly \\
II & From a single RCT & B & Yes, with reservations \\
III & From cohort studies with historical controls or their meta-analyses & C & Uncertainty \\
IV & From retrospective studies such as case-control studies or their meta-analyses & D & No \\
V & Evidence from series of cases without a control group & E & Not advised \\
VI & Opinion of experts in guidelines or consensus & & \\
\hline
\end{tabular}

RCTs: randomized controlled trial. 
Table 2

Virological categories

\begin{tabular}{llll}
\hline & Active carrier & Inactive carrier & Anti-HBc positive (anti-core) \\
\hline HBsAg & Positive & Positive & Negative \\
HBeAg & Positive or negative & Negative & Negative \\
Anti-HBs & Negative & Negative & Positive or negative \\
Anti-HBc & Positive & Positive & Positive \\
HBV DNA serum & $\geq 20,000-2000^{\text {a }} \mathrm{IU} / \mathrm{mL}$ & $<20,000 \mathrm{IU} / \mathrm{mL}$ & Negative $(>90 \%)$ \\
ALT $^{\mathrm{b}}$ & Persistently or intermittently increased & Persistently normal $^{\mathrm{c}}$ & Persistently normal $^{\mathrm{c}}$ \\
HBV DNA tissue $_{\text {Liver damage }^{\mathrm{d}}}$ & Positive & Positive & Positive $^{\mathrm{c}}$ \\
\hline
\end{tabular}

${ }^{\mathrm{a}}$ In anti-HBe positive patients.

b Alanine aminotransferase.

${ }^{c}$ In the absence of other causes of chronic hepatitis and/or of a previous history of chronic hepatitis B.

d Necroinflammatory score $>4$ HAI.

Table 3

Baseline assessment

\begin{tabular}{|c|c|c|c|c|}
\hline \multirow[t]{2}{*}{ Level 1} & \multicolumn{3}{|l|}{ Level 2} & \multirow{2}{*}{$\begin{array}{l}\text { Level } 3 \\
\text { Patients HBV DNA-positive } \\
\text { and/or with chronic hepatitis }\end{array}$} \\
\hline & $\begin{array}{l}\text { Patients with altered } \\
\text { transaminases }\end{array}$ & $\begin{array}{l}\text { Patients HBsAg } \\
\text { positive }\end{array}$ & $\begin{array}{l}\text { Patients anti-core } \\
\text { positive }\end{array}$ & \\
\hline $\begin{array}{l}\text { Transaminase, gamma-glutamyl } \\
\text { transpeptidase, alkaline phosphatase }\end{array}$ & Ultrasonography & HDV & Anti-HBe & IgM anti-HBc (IMx index) \\
\hline Haemochrome & Glycemia & $\mathrm{HBeAg}$, anti-HBe & HBV DNA & Liver biopsy assessment \\
\hline Total and fractionated bilirubin & Cholesterol and triglycerides & HBV DNA & & \\
\hline Anti-HCV & Prothrombin time & & & \\
\hline $\mathrm{HBsAg}$, anti-HBs titre, anti-HBc & Ferritin & & & \\
\hline
\end{tabular}

hepatitis) (activity), as this is frequently associated with progression of liver damage due to HBV (AIII), (4) in all the virological categories (whether or not during prophylaxis or therapy with antivirals), the increase in at least one logarithm of HBV DNA, compared to its nadir, reconfirmed in two consecutive serum tests during monitoring (virologic breakthrough) [4] (AV) Table 2.

\subsection{Clinical definitions}

The assessment of chronic liver disease is the fundamental event of the diagnostic picture (baseline) (AIII) (Table 3) and it requires the use of all the instruments usually utilised in hepatology including, if necessary, trans-cutaneous or transjugular liver biopsy in subjects with coagulation problems (for example patients with blood or kidney diseases).

The baseline diagnosis of the disease is pivotal in the choice of which treatment to adopt, as the risk of severe complications is related to the severity of the underlying liver disease [5].

In order to standardise the definitions the following terms were suggested: (1) infection (not necessarily associated with reactivation of hepatitis) in the case of the detection of $\mathrm{HBV}$ DNA by sensitive HBV assays and/or of HBsAg in patients in whom these markers were originally negative (AVI), (2) reactivation of hepatitis $B$ (hepatitis), in the presence of a significant viremia and ALT levels above the upper normal value (AVI).

\section{Treatment strategies}

The term prophylaxis was used to mean treatment with antiviral drugs of an inactive or occult infection, with the aim of preventing hepatitis reactivation. Prophylaxis was defined as: (1) universal prophylaxis (UP), if it is carried out on the

Table 4

Treatment strategies

\begin{tabular}{|c|c|c|c|c|}
\hline & & \multicolumn{3}{|c|}{ Original virological condition } \\
\hline & & \multirow{2}{*}{$\begin{array}{l}\text { Active carriers } \\
\text { Yes }\end{array}$} & \multicolumn{2}{|c|}{ Inactive carriers or anti-core positive } \\
\hline \multirow[t]{2}{*}{ Clinical condition } & Infection & & Yes & \\
\hline & Hepatitis & Yes & No & \\
\hline \multirow[t]{3}{*}{ Treatment } & & Therapy & Prophylaxis & \\
\hline & & & All the population & Only in patients with infection markers ${ }^{\mathrm{a}}$ \\
\hline & & & Universal & Targeted \\
\hline
\end{tabular}

\footnotetext{
${ }^{a}$ Infection markers: evidence of HBV DNA or HBsAg in serum in originally negative patients.
} 
entire population potentially at risk (inactive carriers and/or anti-core) or targeted prophylaxis (TP), if it is subordinate to the appearance of infection markers (HBV DNA and/or HBsAg) in the absence of hepatitis reactivation (Table 4).

Therapy (T) was understood to mean the treatment of hepatitis B (i.e. chronic hepatitis in active carriers or hepatitis reactivation in previously inactive carriers and in anti-core subjects who develop the seroreversion).

\section{Treatment options}

In Italy, the following drugs are available at present: interferons, either standard or pegylated (both little tolerated in the condition of immunosuppression, especially in transplant patients for the potential risk of rejection) and the nucleos(t)ides analogues (NAs), which include lamivudine and adefovir-dipivoxil for those with HBV monoinfection, with the addition of tenofovir and emtricitabine for patients with $\mathrm{HBV}$-human immunodeficiency virus (HIV) coinfection. Entecavir will be registered in the next months.

Lamivudine, which has a potent antiviral effect, frequently (50-60\% at 4 years) induces the selection of lamivudineresistant mutants in locus YMDD of the polymerase gene (YMDD). Instead adefovir-dipivoxil and entecavir induce a lower selection of mutants in monotherapy (0-3\% at 1-2 year and genotypic resistance in $29 \%$ at 5 years with adefovir in naïve patients; about $10-20 \%$ at 2 years with both drugs in YMDD-carriers) [4-7]. In carriers of the lamivudineresistant variant, the combination of adefovir-dipivoxil and lamivudine can synergically control the selection of the respective mutants [8].

Data from experiences in liver transplanted and HIV patients have shown a relation between the original viremia, the degree of immunosuppression and the selection of mutants during prophylaxis with lamivudine $[9,10]$. Consequently a careful monitoring of the response to treatment and of the resistance is suggested in immunosuppressed patients.

\section{Monitoring}

Once NAs therapy or prophylaxis has been started, monitoring will essentially be through testing serum HBV DNA and ALT levels every 3 months, to assess: (1) response to treatment (i.e. reduction of HBV DNA, preferably below the limit of sensitivity of the amplified techniques and ALT normalization) (BV) and (2) drug-resistance, which should be suspected in the case of virologic breakthrough while ontreatment, in order to activate an early rescue therapy (AIII) [11]. Resistance can be defined clinically by the virologic breakthrough [4] but if available a genotypic testing could be used in order to better define the different mutations and to choose the rescue therapy.

\section{Impact on different specialist fields}

Data regarding hepatitis B in immunocompromised patients are very heterogeneous, so the statements reported here below required an extensive review of the literature and the inclusion of expert opinions where information was lacking. As a result there was a strong indication to promote studies aimed at defining the natural history of hepatitis B in these patients, to assess - also prospectively - different treatment protocols, to promote close cooperation among different specialists and, finally, to constantly update the indications.

Of note, in all fields a pre-immunosuppression assessment (baseline) is recommended, in both HBsAg positive and negative subjects (AIII-V).

\section{Oncology, haematology and haematopoietic stem cell transplantation (HSCT)}

\subsection{Background}

During chemotherapy hepatitis B can make its appearance in two different phases: (1) during the treatment, in relation to the intense bone marrow suppression, which is associated with a strong viral replication and, sometimes, with the emergence of a fulminant hepatitis in the form of fibrosing cholestasis, (2) after the end of therapy, as during the immuno-reconstitution phase the immune response can bring on a reactivation of hepatitis whose clinical course may be more or less severe depending on the baseline condition of the liver and other possible factors that may contribute to the damage.

In oncology, the prevalence of HBsAg positive patients ranges between 5.3\% (in Europe) and 12\% (in China). In these patients, the frequency of clinical HBV reactivation ranges between $20 \%$ and $56 \%$, correlating with the use of steroids, anthracyclines, 5-fluorouracil with some virological indicators (presence of $\mathrm{HBeAg}$ or of e-minus variants and/or of a detectable HBV DNA prior to therapy). The clinical significance of relapse has been clearly associated with the pre-chemotherapy liver function, with a mortality of 5-40\%. The reactivation of hepatitis, moreover, influences the continuation of the chemotherapy, inducing its suspension and not infrequently posing problems of differential diagnosis with regard to drug toxicity. Hepatitis B can develop both in active and in inactive carriers and it is generally associated with the reappearance of a significant viremia in the preceding 2-3 weeks.

In haematology, the frequency of HBsAg positive patients is higher $(12.2 \%$ in Greece and $8.8 \%$ in a recent study from Italy) and the risk of reactivation appears to be greater than in other settings of oncology, depending on the degree of immunosuppression. In this setting, control of the HBV infection assumes great importance in order to prevent HBVrelated complications, but also so as not to modify a highly successful therapeutic schedule. In this field the main prog- 
nostic indicators unfavourably associated with hepatitis B reactivation are, besides those already cited, hyper-transaminasemia and the condition of second or third cycle compared to the first [1,12-14].

In haematology, a $21-67 \%$ (median $50 \%$ ) risk of reactivation has been described, with an average mortality of $20 \%$. In this setting, the available literature is not clear whether the severity of hepatitis in $\mathrm{HBsAg}$ positive patients is directly due to the liver damage caused by $\mathrm{HBV}$ reactivation or by other causes (i.e. veno-occlusive disease (VOD), Graft versus Host Disease (GvHD) or multi organ failure (MOF)) and also the degree of risk in relation to the condition of active or inactive carrier is not clearly determinable.

The risk would appear to be heightened by the use of monoclonal antibodies (anti-CD20, anti-CD52), with the possibility of hepatitis reactivation (even after a cycle of 1-3 months of prophylaxis with lamivudine) at a distance of 12-36 months from the last administration of these drugs, particularly in overt carriers, but also in anti-core subjects. An analogous risk exists in the course of allogeneic HSCT, as the immuno-suppressive effect in the conditioning phase is particularly strong and it is amplified by the subsequent antirejection therapy, so the risk of hepatitis reactivation remains throughout the phase of immuno-reconstitution (in some cases until 1-2 years from transplantation) [1,15-17].

\subsection{Experiences in the different virological categories}

\subsubsection{Active HBsAg-carriers}

In the onco-haematological setting lamivudine therapy of chronic hepatitis in active carriers appears to be effective [1].

\subsubsection{Inactive HBsAg-carriers}

The start of lamivudine therapy at the time of the clinical relapse (hepatitis) in inactive carriers maintains a residual mortality of $20 \%$, probably in relation to the baseline conditions and to the delayed treatment. Instead in retrospective studies lamivudine has been shown to be effective in prophylaxis of hepatitis B (0-9\% of hepatitis reactivation compared to $25-85 \%$ in untreated patients) and in the only prospective study hepatitis relapse developed in $5 \%$ of treated subjects and in $24 \%$ of controls. Moreover, in the study the universal use of lamivudine was better than the TP (activated only at the appearance of HBV DNA with a non-amplified technique, during bimonthly monitoring), both in terms of survival and of hepatitis reactivation $(0 \%$ versus $53 \%, P=0.002)$ $[1,17,18]$.

\subsubsection{Anti-core patients (HBsAg negative)}

In the oncological setting, there are no data, at present, for this virological category, which can reach $20-40 \%$ in averagely endemic areas and $70-80 \%$ in highly endemic areas. However, in the haematological setting, out of a total of 176 patients described in literature, seroreversion has been reported in 21 subjects (12\%) during conventional chemotherapy, whether or not this was associated with
HSCT, with percentages of 4-30\% during chemotherapy and 14-50\% in the course of autologous transplantation.

After autologous HSCT hepatitis B developed in anticore patients later (6-52 months, average 19 months) than in overt carriers (average 2-3 months) and none of the patients described died of hepatitis B (in seven cases during therapy with lamivudine, started at the time of the clinical relapse). After the reactivation 9 of the 10 patients remained HBsAg positive and 1 lost the HBsAg during follow-up. Instead, 2 deaths out of 39 subjects with seroreversion have been reported in literature after allogeneic HSCT and this appeared to have been significantly linked to the absence of protective antibodies (anti-HBs) in the donor and to GvHD [1].

Recently the introduction in haematologic treatments of monoclonal antilymphocyte B and T antibodies (anti-CD20 and anti-CD52), used alone or together with chemotherapy, has been associated with the signalling of six cases of seroreversion in anti-core subjects, in three cases with a fulminant form and death of the patients, despite therapy with lamivudine [1].

\subsection{Statements}

1. In active carriers, therapy is considered useful to control the liver disease pre- and post-immunosuppressive treatments. In HSCT, in particular, the control of the HBV-related disease permits a more precise diagnosis and treatment of specific liver complications (GvHD and VOD). In these patients, antiviral therapy should be continued lifelong (due to the high risk of relapse after withdrawal) or at least until the disappearance of $\mathrm{HBsAg}$ in serum. A strict monitoring of mutants should be activated, in order to prevent hepatitis relapse with rescue therapy (AIII).

2. In the inactive carriers, $U P$ appears to be indicated and should be continued for the entire phase of chemotherapy, until at least 6-12 months after the end of the treatment [18] (BV). The optimal duration of the prophylaxis is still debated and requires prospective studies. In any case, the panel recommends monitoring of the viremia after suspension, for the prompt diagnosis and return to treatment in the case of reactivation.

3. In anti-core (HBsAg negative) patients, two different strategies can be identified:

(a) In oncology or in patients undergoing mild haematological therapies (judged to be at low immunosuppressive potential, such as the ABVD of the CHOP 21 days scheme), HBsAg monitoring every 1-3 months is advised, with the activation of TP or therapy in the case of seroreversion or hepatitis reactivation, respectively (BVI). Instead the use of HBV DNA monitoring for targeted prophylaxis remains controversial, because of the lack of data referred to the timing and duration of the monitoring and to the clinical significance of minimal levels of detectable 
viremia (i.e. the presence of low levels of serum $\mathrm{HBV}$ DNA in anti-core patients after solid organs transplantation is not constantly associated with hepatitis relapse) [19] (CV).

(b) In subjects who need to be treated with intense immunosuppression (chemotherapy with fludarabine, dose-sense regimes, allogeneic transplant, autologous myeloablative transplant, induction in acute leukaemia, use of monoclonal antibodies) UP is proposed. This approach is strongly indicated in the haematological setting and in patients with signs of a chronic hepatitis (due to a previous history of HBVrelated disease and/or to other causes of chronic hepatitis) and/or with a positive serum HBV DNA and/or positive for anti-HBe antibodies at the baseline evaluation [20] (CVI). In anti-core subjects, clinical studies are recommended in the future.

\subsection{Effects of different virological conditions in donors \\ $(D)$ and recipients $(R)$ of allogeneic-HSCT}

1. D (HBsAg-/anti-HBs+/anti-HBc \pm ) $\rightarrow \mathrm{R}(\mathrm{HBsAg}+)$. In the case of transplant from an immunized (anti-HBs positive) donor to an overt carrier (HBsAg positive) recipient, two possible scenarios have been described: (a) the chance of adoptive transfer of immunity with the possible clearance of $\mathrm{HBsAg}$ (especially if recipients are treated with lamivudine), (b) an acute and sometimes fulminant hepatitis (in historical series) [1].

2. $\mathrm{D}$ (HBsAg-/anti-HBs \pm /anti-core +$) \rightarrow \mathrm{R} \quad$ (HBsAg-/anti$\mathrm{HBs} \pm$ /anti-core \pm ). Only few data are available, indicating that in the case of transplant from an anti-core donor the risk of seroreversion in the recipient would appear to be negligible in anti-core positive recipients and greater in the case of complete negativity for the markers of previous contact with HBV (nä̈ve), in analogy with what has been described in the transplant of solid organs [21].

3. $\mathrm{D}(\mathrm{HBsAg}+) \rightarrow \mathrm{R}$ (HBsAg-). In a few studies, transplant from HBsAg positive donor was associated with hepatitis in $44-62 \%$ of recipients, with generic hepatic mortality in $33-75 \%$ of cases, although the role of HBV in these clinical events was not well defined. In a historical retrospective multicentre study performed in the pre-antiviral phase, the anti-HBV specific immunoglobulins (HBIG) were not protective against the transmission of the infection. In contrast, in a recent study the activation of therapy with lamivudine in donors and of prophylaxis with the same antiviral in recipients significantly reduced the HBVrelated hepatitis rate $(48 \%$ versus $7 \%, P=0.002)$ and mortality $(24 \%$ versus $0 \%, P=0.01)$ compared to a historical control group [1].

\subsection{General statements in HSCT}

The panel of experts who met in Turin proposed the following indications in HSCT, if compatible with the timing of the ongoing treatment:

a. Vaccination of the recipient prior to transplant with accelerated protocols (recombinant vaccine $40 \mu \mathrm{g}$ by intramuscular route time $0-1-2$ months or 0-7-21 days), especially if he/she is naïve (BIII).

b. Vaccination of the donor not immunized prior to transplant, with accelerated protocols (recombinant vaccine $20 \mu \mathrm{g}$ by intramuscular route time $0-1-2$ months or 0-7-21 days), in the case of allogeneic HSCT (AV).

c. Preferential allocation of anti-core organs to vaccinated recipients or those with markers of prior contact with $\mathrm{HBV}$ (BV).

d. Treatment of the HBsAg positive donor with lamivudine pre-HSCT in order to reduce infectivity through the reduction of viremia (preferably below the limit of sensitivity of an amplified assay) and universal prophylaxis of the recipient on the day before the transplant (AIV).

e. The use of high doses of HBIG (intravenous 10,000 IU) during infusion of hematopoietic stem cells from overt carriers (who have been preventively treated with antivirals) in HBsAg negative recipients. There is a lack of data about the neutralising effect of HBIG in this setting in the antivirals era, but while awaiting prospective studies there has been recent evidence in the liver transplantation setting of a direct correlation between HBV DNA in serum (now preventively reduced in $\mathrm{HBsAg}$ positive recipients by antivirals) and the neutralizing power of the immunoglobulins [22] (BVI).

The panel agrees that because of the actual results of the hepatologic and haematologic therapy there is no reason to deny a HSCT from a HBV positive donor (any form) if the risk-benefit ratio is in favour of transplantation. Moreover in the case of a HLA identical family HBV positive member there is no point in wasting time and resources in searching for an unrelated donor in the international bone marrow donor bank.

\section{Dialysis and solid organs transplants (kidney, heart and lung)}

\subsection{Background}

\subsubsection{Dialysis}

The incidence of overt carriers of HBsAg among dialysed patients is $0-7 \%$ in developed countries and $10-20 \%$ in developing ones. In these subjects, the frequent normality of the transaminase makes clinical judgment difficult, confirming the fundamental role of the virological markers (quantitative HBV DNA) and of the liver biopsy to distinguish between active and inactive carriers (baseline). In this setting, data about the condition of occult carrier among anti-core patients are scarce and regard the sole presence of viremia in serum, whose diagnostic sensitivity is low. 
In kidney transplant, the condition of HBsAg carrier can be estimated in 10-20\% of cases and it is associated with a significantly higher risk of death (OR 2.49, 95\% CI), independent of the viremic condition (active or inactive carrier), and the chronic hepatitis presents an accelerated course towards cirrhosis (5.3-12\%-year), decompensation and hepatocarcinoma $[23,24]$.

In heart and lung transplant, Italian reports have signalled HBsAg positivity in $2.3-3.7 \%$ of recipients. In this setting, the evolution of the HBV-related disease is accelerated in active carriers and the risk of hepatitis $B$ reactivation posttransplant is over $50 \%$ in originally inactive subjects. Finally, the risk of seroreversion post-surgery (de novo hepatitis B) in HBsAg negative/anti-core recipients seems to be lower than $5 \%$ [25-27].

\subsection{Clinical experiences in nephrology}

No controlled trials for the treatment of HBV with either interferon or lamivudine in dialysed patients or in kidney transplants are currently available. Interferon can be used to treat dialysed patients with chronic hepatitis B, but it is contraindicated in transplanted patients. Short-term administration of lamivudine monotherapy is effective but when the drug is withdrawn, viremia rebounds and hepatitis relapses in most cases. Continuous administration of lamivudine monotherapy for 3-4 years is able to obtain long-term suppression of HBV replication and may prevent the development of liver related complications and mortality [28]. Secondary treatment failure is caused by the emergence of YMDD which in some patients herald hepatitic flares and progression of the liver disease.

\subsection{Statements in relation to transplant recipients}

\subsubsection{Active carrier}

In candidates for kidney, heart or lung transplant the indication to therapy is confirmed, both in the pre-transplant (with NAs or interferons, when they are tolerated) (BV) and in the post-transplant phase (only NAs in view of the high risk of interferon-induced rejection) (AV).

\subsubsection{Inactive carrier}

Pre-transplant and during dialysis there is no indication for prophylaxis but biochemical and virological monitoring is advised, if the diagnosis has been confirmed by strict adherence to previously defined criteria. Instead, therapy should be used in the re-activated forms (HBV DNA $\geq 20,000 \mathrm{IU} / \mathrm{mL}$ ), especially if associated with significant liver damage (HAI $>4$ and/or signs of fibrotic disease by noninvasive methods) (BVI). Post-transplant, instead, there is an indication to $U P$, in relation to the available data on mortality in HBV carriers, independently from their virological condition [23] (BV).

\subsubsection{Anti-core recipient}

In HBsAg negative and anti-core positive recipients of kidney, heart and lung transplant the presence of subclinical manifestations (low levels of circulating HBV DNA detectable with amplified techniques post-transplant) without seroreversion in over $95 \%$ of cases $[19,23,24,27]$ has been signalled. In this condition, only monitoring of the HBsAg is required, with the activation of TP or therapy only in the case of seroreversion and/or hepatitis, respectively (BV).

\subsection{Statements in relation to transplant donors}

\subsubsection{Anti-core donors}

In the case of kidney, heart or lung allocation from an HBsAg negative/anti-core positive/anti-HBs positive or negative donor in a HBsAg negative recipient, the risk of hepatitis $\mathrm{B}$ appears to be less than 5\% [27,29]. The low risk does not justify preventive prophylaxis, but only HBsAg monitoring (every 3-6 months and/or in the case of transaminase increase) and the use of targeted prophylaxis or therapy only in the case of seroreversion (BIV). In analogy to what has been signalled before the risk connected with the use of anticore positive organs is further reduced by the indications reported in Section 7.5 (AVI).

\subsubsection{HBsAg positive donors}

In this condition, the risk of transmission of the HBV infection is very high in the absence of prophylaxis, especially from $\mathrm{HBeAg}$ positive donors [30]. Recently a report has signalled the post-transplant control of hepatitis B in HBsAg negative/anti-HBs positive recipients of kidneys from $\mathrm{HBs} A g$ positive donors ( $\mathrm{HBeAg}$ and $\mathrm{HBV}$ DNAnegative) while on lamivudine prophylaxis [31]. In Italy, the use of these organs is controlled by national guidelines (www.governo.it/GovernoInforma/Dossier/donatori_organi/ linee_guida.html).

\section{Liver transplantation}

\subsection{Background}

The risk of post-transplantation hepatitis B is strictly influenced from both recipient and donor virological characteristics:

(a) HBsAg positive recipients: in the absence of preand post-operative prophylaxis the risk of posttransplantation hepatitis B is over $80 \%$. In this condition, the use of antivirals before transplant (one single antiviral in the case of wild type virus, combined with a second one that is active on the mutants, in the condition of drug resistance with active replication), associated with HBIG after surgery (combined prophylaxis), is protective in more than $90 \%$ of patients $[32,33]$. 
(b) HBsAg negative and anti-core positive recipients: in absence of prophylaxis the risk of seroreversion after transplantation (de-novo hepatitis B) is less than 5\% from naïve liver donors and $10-15 \%$ from anti-core positive donors [19,34].

(c) HBsAg positive donors: the risk of hepatitis $\mathrm{B}$ transmission with a liver from an HBsAg positive donor is high, as the neutralising effect of HBIG is very low in this setting and the reappearance of HDV, in coinfected recipients, is constant. In this particular condition, the reactivation of hepatitis would appear to be controlled by the combination of two antivirals in the long term [35].

In Italy, the transplantation is controlled by the national guidelines (www.governo.it/GovernoInforma/Dossier/ donatori_organi/linee_guida.html).

(d) HBsAg negative/anti-core positive donors: in this category the overall risk of HBV transmission and hepatitis is high (33-78\%), in the absence of prophylaxis, ranging from $70 \%$ in naïve to $10-15 \%$ in anti-core recipients. Combined prophylaxis with lamivudine and HBIG controls relapse in nearly all cases, while personalised prophylaxis with only HBIG or only lamivudine has been suggested in low-risk recipients (anti-core positive) [34]. Comparative studies are not available in this setting.

\subsection{Statements in relation to recipients}

In all $\mathrm{HBsAg}$ positive carriers, there is an indication to $U P$ post-surgery according to their original virological condition (AIII):

(a) In active carriers, therapy before surgery is indicated (with one or two antivirals in cases of YMDD mutants), with the aim of achieving the reduction of HBV DNA below the limit of sensitive HBV assays (AIII) or at least below $<20,000 \mathrm{IU} / \mathrm{mL}$ (BIII), in association with combined prophylaxis (HBIG and one or two antivirals, as previously reported) in the post-operative period.

(b) In inactive carriers, the role of therapy before surgery remains controversial (BIV) because of the high (>80\%) protective effect of post-transplantation combined prophylaxis (BIII). In these subjects, a preventive reduction of HBV DNA before surgery might not be necessary, with regard to the minimal residual risk, but it could be desirable in order to save HBIG in the long term after liver transplantation (AV). Likewise, in subjects with spontaneous undetectable viremia (PCR-negative) or with levels around the limit of detectability $(<2000 \mathrm{IU} / \mathrm{mL})$, especially if coinfected with HDV, the protective power of just HBIG seems to be very high (AIII). Although also in this condition the use of the combined prophylaxis after liver transplantation permits a considerable saving of HBIG in the long term (AV). (c) In HBsAg negative/anti-core positive recipients, in analogy with what has been described in the other transplants, albeit in the presence of serum and intra-hepatic evidence of reinfection by HBV in the post-transplant period, the risk of seroreversion is practically nil $[36,37]$ and so there is no indication for any prophylaxis, but only for the monitoring of HBsAg.

\subsection{Statements in relation to donors}

As indicated by the national guidelines the use of organs from HBsAg positive donors should be considered only in conditions of emergency, avoiding their use in HDV recipients. The use of $U P$ with two antivirals post-transplant could permit the control of hepatitis $B$ recurrence in the long term (BV). Instead the use of livers from HBsAg negative/anticore positive donors justifies the adherence to the indications reported in the paragraph 7.5 and the activation of $U P$ with HBIG and lamivudine in the case of allocation to naïve recipients (BV) and with only HBIG or only lamivudine (after the administration of HBIG in the peri-operative period) in anti-core recipients $(\mathrm{BV})$.

\section{Rheumatology}

\subsection{Background}

Reports regarding the reactivation of $\mathrm{HBV}$ in the rheumatology setting are episodic, during the course of hydroxychloroquine, azathioprine, methotrexate and tumour necrosis factor (TNF) inhibitors. The few data available all refer to active and inactive HBsAg- carriers. Instead reports on anti-CD20 derive from haematological experience, and like in haematology the risk of HBV reactivation in the rheumatology setting would appear to be linked both to the phase of immuno-suppression and to that of immunoreconstitution. In the meantime no reactivations have been reported in the few HBsAg positive rheumatology patients undergoing UP with lamivudine during immunosuppressive therapy [38-41].

In the absence of data, the expert panel has identified two risk categories with regard to the type and to the degree of immunosuppression: (a) high risk of $H B V$ reactivation in patients undergoing the following therapy: anti-TNF antibodies, medium to high dosage steroids $(>7.5 \mathrm{mg} / \mathrm{die})$ for prolonged periods [42], immunosuppressors such as cyclophosphamide, methotrexate, leflunomide, calcineurin antagonists, azathioprine and mycophenolate mofetil. Although cases of viral reactivation have not yet been described in rheumatology patients undergoing treatment with anti-CD20 antibodies, the data which has emerged in other specialist circles suggest the inclusion in this group of these and other monoclonals (BVI); (b) low risk of $H B V$ reactivation in patients treated with steroids at $<7.5 \mathrm{mg} / \mathrm{die}$, sulfasalazine and hydroxychloroquine (BVI). 


\subsection{Statements}

Among HBsAg positive patients therapy is indicated in active carriers (AVI) and UP with NAs is suggested in inactive carriers who underwent high-risk treatments, especially if they are subjects with manifestations of chronic liver disease due to the previous activity of HBV or other causes (BVI). Finally, in inactive HBsAg-carriers treated with low-risk therapies and in HBsAg negative/anti-core positive subjects the proposal is a strategy of monitoring, with the activation of therapy or TP in the case of viral reactivation (HBV DNA $\geq 20,000 \mathrm{IU} / \mathrm{mL}$ ) or seroreversion, respectively (BVI).

Prophylaxis should be started $2-4$ weeks before the immunosuppressive therapy if possible and continued for at least 6-12 months afterwards (i.e. after immunosuppressive therapy has been suspended). Haematology literature advises particular caution in suspending prophylaxis, especially in subjects treated with repeated cycles of monoclonal antibodies.

\subsection{Peculiar conditions in the rheumatology setting}

\subsubsection{Anti-HBV vaccination}

Vaccination in rheumatology patients remains controversial and its cost/benefit ratio should be carefully assessed in groups particularly at risk of $\mathrm{HBV}$ (for example those living with HBsAg positive individuals or health workers).

\subsubsection{Panarteritis nodosa (PAN)}

This is a rare necrotising vasculitis that interests small and medium-sized arteries which presents, at least in a portion of cases, a pathogenic correlation with HBV infection. In the treatment of HBV-related PAN, the immunosuppressive therapy (which also poses the question of an uncontrolled activation of the virus) should be associated with an antiviral therapy (in active carriers) or UP (in inactive carriers) to repress viral replication. In this regard, single cases and observational studies with small numbers of cases have documented the efficacy of interferon (IFN) and lamivudine $(\mathrm{AV})$.

\section{HIV}

\subsection{Background}

The indications in this setting, refer to the recently published European Association for the Study of the Liver (EASL) guidelines. Cirrhosis and liver cancer are the second cause of death worldwide in HIV carriers (3-4 million), $9 \%$ of whom have HBV infection. Coinfection with HIV increases the rate of chronic HBV infection, reduces the annual rate of seroconversion to anti-HBe and to anti-HBs and it may be linked to the reactivation of the occult infec- tion in HBsAg negative subjects in the presence of severe immuno-depletion. Moreover, co-infection with HIV accelerates progression towards cirrhosis and liver decompensation and reduces survival in decompensated cirrhotics. Therefore, mortality due to liver disease in those co-infected with HIV$\mathrm{HBV}$ is higher compared to subjects with just HBV infection $[43,44]$.

\subsection{Statements}

\subsubsection{Patients undergoing antiretroviral viral therapy (ART)}

In active and inactive carriers, therapy and $U P$ with antivirals (utilising the same nucleos(t)ides effective on HBV used in the treatment of HIV infection) are indicated, respectively (AIII).

In HBsAg negative/anti-core positive subjects, the condition of occult carrier, characterised by HBV DNA positivity in serum and/or in the liver, has been identified in 35-90\% of subjects with HIV coinfection using high sensitivity techniques, and only in $1 \%$ of cases with less sensitive techniques. Even in the presence of anecdotal reports of reactivation during immunodepletion and/or of suspension of lamivudine, the risk of seroreversion appears to be very low $(0.23 / 100$ patients/year) and it does not therefore justify any prophylaxis but only monitoring [44] (BVI).

\subsubsection{Patients who do not require ART}

In active carriers, therapy with interferons or antivirals is indicated. In these subjects, treatment should preferably be administered using drugs which do not have any effect on HIV and which do not, in the future, induce resistance to ART (interferons, entecavir, telbivudine) (AIII).

Instead, in inactive carriers and in anti-core subjects monitoring of HBV DNA or HBsAg, respectively, is recommended, with activation of therapy or TP in the case of seroreversion (BVI).

\section{Conclusions}

The literature on hepatitis $\mathrm{B}$ in immunosuppressed patients is heterogeneous. It refers mainly to the pre-analogue nucleos(t)ides era and the period prior to the introduction of the modern techniques of determination and quantification of the viremia, which raises many doubts and difficulties about the interpretation of the studies and leaves several aspects still a matter of debate. This encourages the proposal of a network of communication between different specialists involved, in order to better define the natural history, the potential risk of hepatitis B and the results of the various strategies proposed.

Even in the light of such premises today it appears to be justified to propose a rational approach to the problem of hepatitis B in immunocompromised patients (Table 5), which 
Table 5

Prophylaxis and therapy in immunocompromised subjects

\begin{tabular}{|c|c|c|c|}
\hline & $\begin{array}{l}\text { Active carrier } \\
\text { HBsAg positive }\end{array}$ & Inactive carrier & $\begin{array}{l}\text { Anti-core } \\
\text { HBsAg negative }\end{array}$ \\
\hline Haematology and HSCT & $\mathrm{T}$ & UP & $\begin{array}{l}\text { UP }{ }^{a} \text { High risk } \\
\text { Monitoring low risk }\end{array}$ \\
\hline Oncology & $\mathrm{T}$ & UP & Monitoring \\
\hline Solid organ transplant & $\mathrm{T}$ & UP & Monitoring \\
\hline Nephrology (dialysis) & $\mathrm{T}$ & Monitoring & Monitoring \\
\hline Rheumatology & $\mathrm{T}$ & $\begin{array}{l}\mathrm{UP}(\text { high risk) } \\
\text { Monitoring }(\text { low risk) }\end{array}$ & Monitoring \\
\hline HIV & $\mathrm{T}(\mathrm{ART}-$ and ART+) & $\begin{array}{l}\text { Monitoring (ART-) } \\
\text { UP (ART+) }\end{array}$ & Monitoring \\
\hline Liver transplantation & $\mathrm{T}^{\mathrm{d}}($ pre-LT $) \rightarrow \mathrm{UP}^{\mathrm{d}}($ post-LT $)$ & $\mathrm{UP}^{\mathrm{d}, \mathrm{e}}($ pre-LT if $\mathrm{PCR}+) \rightarrow \mathrm{UP}^{\mathrm{d}, \mathrm{e}}$ post-LT & Monitoring \\
\hline
\end{tabular}

ART: antiretroviral therapy, T: therapy, UP: universal prophylaxis, TP: targeted prophylaxis, Monitoring: periodic checking of marker of infection in order to activate targeted prophylaxis or therapy in anti-core or inactive carriers, respectively, with signs of infection and/or hepatitis B reactivation.

a High risk: chemotherapy with fludarabine, dose-sense regimes, allogeneic transplant, autologous myeloablative transplant, induction in acute leukaemia, use of monoclonal antibodies (anti-CD20, anti-CD52).

b Immunosuppression therapy (high risk): antiTNF antibodies, medium to high dosage steroids ( $>7.5 \mathrm{mg} / \mathrm{die}$ ) for prolonged periods, cyclophosphamide, methotrexate, leflunomide, calcineurin antagonists, azathioprine and mycophenolate mofetil.

${ }^{\mathrm{c}}$ Immunosuppression therapy (low risk): steroids $<7.5 \mathrm{mg} / \mathrm{die}$, sulfasalazine and hydroxychloroquine.

${ }^{\mathrm{d}}$ Lamivudine in naïve, associated with adefovir-dipivoxil in lamivudine-resistant patients.

e Anti-hepatitis B immunoglobulis (HBIG).

provides for:

- monitoring the markers of the HBV in all subjects starting immunosuppressive therapies and the evaluation of their liver condition (baseline);

- therapy of active carriers;

- prophylaxis of inactive carriers, especially if they are undergoing immunosuppressive therapies judged to be at high risk. In this case, the treatment should be limited to the period of immunosuppression and to the subsequent reconstitution, in the settings in which this is possible (haematology and HSCT, rheumatology, oncology, HIV) and continued indefinitely in the condition of solid organs transplantation;

- biochemical and HBsAg monitoring (or viremia monitoring, when this strategy is chosen) with the aim of activating a swift therapy or a targeted prophylaxis, respectively in inactive carriers and in anti-core subjects not undergoing prophylaxis;

- evaluation, in the transplant setting, of the virological characteristics of both recipients and donors, in order to activate the best prophylactic strategies and to obtain the best allocation in the case of a significant risk of hepatitis B after surgery (organs from donors who are positive for HBsAg or anti-core antibodies).

Finally, the greatest uncertainty regards the most costeffective management of HBsAg negative/anti-HBc positive (anti-core) subjects. For most of these patients involved in the different settings, a simple monitoring of HBsAg seems to be the most rational approach. In contrast, in those enlisted for HSCT and/or undergoing intense chemotherapy, especially in the presence of multicycles of anti-CD20 or anti-CD52 monoclonal antibodies, UP is considered to be cost-effective and is therefore proposed. However, clinical studies in this setting are scarce and there is an absolute need for these to be conducted in the near future.

\section{Practice points}

- Evaluation of liver condition (baseline) and monitoring of HBV markers and viremia in all subjects starting immunosuppressive therapies and/or treated with antivirals for prophylaxis or therapy of hepatitis B.

- Therapy of active carriers.

- Prophylaxis of inactive carriers and of anticore patients in the haematologic setting, especially if they are undergoing immunosuppressive therapies judged to be at high risk.

- Monitoring of HBV markers in anti-core subjects not undergoing prophylaxis.

- Evaluation, in the transplant setting, of the virological characteristics of both recipients and donors, in order to activate the best prophylactic strategies and to obtain the best allocation in the case of a significant risk of hepatitis $B$ after surgery. 


\section{Research agenda}

- Clinical relevance of hepatitis B in overt and occult (anti-core) HBV carriers undergoing immunosuppressive therapies.

- Clinical efficacy and relevance of prophylaxis, therapy or HBsAg monitoring in the different virological categories and specialist fields.

- Role of mono- or combined-therapy with different antivirals in patients undergoing immunosuppressive therapies in terms of mutants selection or clinical and virologic response.

\section{Conflict of interest statement}

None declared.

\section{List of abbreviations}

ABVD, Doxorubicine, Bleomycin, Vinblastine, Dacarbazine (standard therapy for Hodgkin lymphoma); anti-core, an HBsAg negative/anti-HBc positive subject; anti-HBc, antibody to hepatitis B core antigen; anti-HBe, antibody to hepatitis e antigen; anti-HBs, antibody to HBsAg; ART, anti-retroviral therapy; CHOP, Cyclophosphamide, Doxorubicin, Vincristine, Prednisolone; D, donor; GvHD, Graft versus Host Disease; HAI, histology activity index; HBeAg, hepatitis $\mathrm{B}$ envelope antigen; HBIG, anti-HBV specific immunoglobulins; HBsAg, hepatitis B surface antigen; $\mathrm{HBV}$, hepatitis B virus; $\mathrm{HCV}$, hepatitis $\mathrm{C}$ virus; $\mathrm{HDV}$, hepatitis D virus; HIV, human immunodeficiency virus; HSCT, haematopoietic stem cells transplantation; IFN, interferon; INR, international normal ratio; MOF, multi organ failure; NAs, nucleos(t)ides analogues; PCR, polymerase chain reaction; PNLG, Piano Nazionale Linee Guida (National Guidelines); R, recipient; RCTs, randomized controlled trials; T, therapy; TNF, tumor necrosis factor; TP, targeted prophylaxis; UP, universal prophylaxis; US, ultrasound; VOD, veno-occlusive disease; YMDD, lamivudine-resistant mutants in locus YMDD of the polymerase gene.

\section{Acknowledgements}

To Mrs. Susan Phillips for the language revision and to Mrs. Monica Moretti, Mrs. Clara Grossi and Silvia Carenzi, $\mathrm{MD}$, for their precious assistance in the preparation of the first AISF Single Topic conference and of the manuscript. We want to thank all the scientific associations who endorsed the meeting and the participants in the different groups of discussion and in the plenary session.

\section{Appendix A}

The First AISF Single Topic was endorsed by: Società Italiana di Malattie Infettive e Tropicali (SIMIT), Associazione Italiana di Oncologia Medica (AIOM), Società Italiana di Ematologia (SIE), Società Italiana di Nefrologia (SIN), Società Italiana di Reumatologia (SIR), Società Italiana di Trapianti d'Organo (SITO).

\section{References}

[1] Yeo W, Johnson PJ. Diagnosis, prevention and management of hepatitis B virus reactivation during anticancer therapy. Hepatology 2006;43:209-20.

[2] Raimondo G, Pollicino T, Cacciolla I, Squadrito G. Occult hepatitis B infection. J Hepatol 2007;46(1):160-70.

[3] Consensus statement of EASL International Consensus Conference on Hepatitis B. J Hepatol 2003;38:533-40.

[4] Keeffe EB, Dieterich DT, Han SHB, Jacobson IM, Martin P, Schiff ER, et al. A treatment algorithm for the management of chronic hepatitis $B$ virus infection in the United States: an update. Clin Gastroenterol Hepatol 2006;4:936-62.

[5] Di Marco V, Marzano A, Lampertico P, Andreone P, Santantonio T, Almasio PL, et al. Clinical outcome of HBeAg-negative chronic hepatitis $\mathrm{B}$ in relation to virological response to lamivudine. Hepatology 2004;40:883-91.

[6] Fung SK, Chae HB, Fontana RJ, Conjeevaram H, Marrero J, Oberhelman K, et al. Virologic response and resistance to adefovir in patients with chronic hepatitis. J Hepatol 2006;44:283-90.

[7] Locarnini S, Hatzakis A, Heathcote J, Keeffe EB, Liang TJ, Mutimer $\mathrm{D}$, et al. Management of antiviral resistance in patients with chronic hepatitis B. Antivir Ther 2004;9:679-93.

[8] Mutimer D. Adefovir-lamivudine combination therapy and hepatitis B kinetics. J Hepatol 2005;43:200-2.

[9] Mutimer D, Dusheiko G, Barrett C, Grellier L, Ahmed M, Anschuetz $\mathrm{G}$, et al. Lamivudine without HBIg for prevention of graft reinfection by hepatitis B: long-term follow-up. Transplantation 2000;70: 809-15.

[10] Matthews GV, Bartholomeusz A, Locarnini S, Ayres A, Sasaduesz J, Seaberg E, et al. Characteristics of drug resistant $\mathrm{HBV}$ in an international collaborative study of HIV-HBV-infected individuals on extended lamivudine therapy. AIDS 2006;20:863-70.

[11] Lampertico P, Vigano M, Manenti E, Iavarone M, Lunghi G, Colombo M. Adefovir rapidly suppresses hepatitis $B$ in HBeAg-negative patients developing genotypic resistance to lamivudine. Hepatology 2005;42:1414-9.

[12] Alexopoulos CG, Vaslamatzis M, Hatzidimitriou G. Prevalence of hepatitis B virus marker positivity and evolution of hepatitis B virus profile, during chemotherapy, in patients with solid tumours. Br J Cancer 1999;81:69-74.

[13] Marcucci F, Mele A, Spada E, Candido A, Bianco E, Pulsoni A, et al. High prevalence of hepatitis B virus infection in B-cell non-Hodgkin's lymphoma. Haematologica 2006;91:554-7.

[14] Takai S, Tsurumi H, Ando K, Kasahara S, Sawada M, Yamada T, et al. Prevalence of hepatitis $\mathrm{B}$ and $\mathrm{C}$ virus infection in haematological malignancies and liver injury following chemotherapy. Eur J Hematol 2005;74:158-65.

[15] Dai MS, Chao TY, Kao WY, Shyu RY, Liu TM. Delayed hepatitis $\mathrm{B}$ virus reactivation after cessation of pre-emptive lamivudine in 
lymphoma patients treated with rituximab plus CHOP. Ann Hematol 2004;83:769-74.

[16] Hui CK, Cheung WW, Au WY, Lie AK, Zhang HY, Yueng YH, et al. Hepatitis B reactivation after withdrawal of pre-emptive lamivudine in patients with haematological malignancy on completion of cytotoxic chemotherapy. Gut 2005;54:1597-603.

[17] Lau GK, Yiu HH, Fong DY, Cheng HC, Au WY, Lai LS, et al. Early is superior to deferred pre-emptive lamivudine therapy for hepatitis B patients undergoing chemotherapy. Gastroenterology 2003;125:1742-9.

[18] Kohrt HE, Ouyang DL, Keeffe EB. Systemic review: lamivudine prophylaxis for chemotherapy-induced reactivation of chronic hepatitis $B$ virus infection. Aliment Pharmacol Ther 2006;24:1003-6.

[19] Knoll A, Pietrzyk M, Loss M, Goetz WA, Jilg W. Solid organ transplantation in HBsAg-negative patients with antibodies to HBV core antigen: low risk of HBV reactivation. Transplantation 2005;79: 1631-3.

[20] Allain JP. Occult hepatitis B virus infection: implications in transfusion. Vox Sang 2004;86:83-91.

[21] Zekri AR, Mohamed WS, Samra MA, Sherif GM, El-Shehaby AM, El-Sayed MH. Risk factors for cytomegalovirus, hepatitis B and C virus reactivation after bone marrow transplantation. Transpl Immunol 2004;13:305-11.

[22] Dickson RC, Terrault NA, Ishitani M, Reddy KR, Sheiner P, Luketic $\mathrm{V}$, et al. Protective antibody levels and dose requirements for IV 5\% Nabi Hepatitis B immune globulin combined with lamivudine in liver transplantation for hepatitis B-induced end stage liver disease. Liver Transpl 2006;12:124-33.

[23] Fabrizi F, Martin P, Dixit V, Kanwal F, Dulai G. HBsAg seropositive status and survival after renal transplantation: meta-analysis of observational studies. Am J Transplant 2005;5:2913-21.

[24] Berger A, Preiser W, Kachel HG, Sturmer M, Doerr HW. HBV reactivation after kidney transplantation. J Clin Virol 2005;32:162-5.

[25] Fagiuoli S, Minniti F, Pevere S, Farinati F, Burra P, Livi U, et al. $\mathrm{HBV}$ and $\mathrm{HCV}$ infections in heart transplant recipients. J Heart Lung Transplant 2001;20:718-24.

[26] Zampino R, Marrone A, Ragone E, Costagliola L, Cirillo G, Karayiannis $\mathrm{P}$, et al. Heart transplantation in patients with chronic hepatitis B:clinical evaluation, molecular analysis and effect of treatment. Transplantation 2005;80:1340-3.

[27] De Feo TM, Grossi P, Poli F, Mozzi F, Messa P, Minetti E, et al. Kidney transplantation from anti-HBc positive donors: results from a retrospective Italian study. Transplantation 2006;81:76-80.

[28] Fabrizi F, Dulai G, Dixit V, Bunnapradist S, Martin P. Lamivudine for the treatment of hepatitis B virus-related liver disease after renal transplantation: meta-analysis of clinical trials. Transplantation 2004;77:859-64.

[29] Fabrizi F, Lunghi G, Martin P. Hepatitis B virus infection in hemodialysis: recent discoveries. J Nephrol 2002;15:463-8.
[30] Natov SN, Pereira BJ. Transmission of viral hepatitis by kidney transplantation. Transplant Infect Dis 2002;4:117-23.

[31] Berber I, Aydin C, Yigit F, Turkmen F, Titiz I, Altaca G. The effect of HBsAg-positivity of kidney donors on long-term patient and graft outcome. Transplant Proc 2005;37:4173-5.

[32] Marzano A, Lampertico P, Mazzaferro V, Carenzi S, Vigano M, Romito $\mathrm{R}$, et al. Prophylaxis of hepatitis B virus recurrence after liver transplantation in carriers of lamivudine-resistant mutants. Liver Transpl 2005;11:532-8.

[33] Marzano A, Gaia S, Ghisetti V, Carenzi S, Premoli A, DebernardiVenon W, et al. Viral load at the time of liver transplantation and risk of hepatitis B virus recurrence. Liver Transpl 2005;11:402-9.

[34] Manzarbeitia C, Reich DJ, Ortiz JA, Rothstein KD, Araya VR, Munoz SJ. Safe use of livers from donors with positive hepatitis B core antibody. Liver Transpl 2002;8:556-61.

[35] Franchello A, Ghisetti V, Marzano A, Romagnoli R, Salizzoni M. Transplantation of hepatitis B surface antigen-positive livers into hepatitis B virus-positive recipients and the role of hepatitis delta coinfection. Liver Transpl 2005;11:922-8.

[36] Ghisetti V, Marzano A, Zamboni F, Barbui A, Franchello A, Gaia S, et al. Occult hepatitis B virus infection in HBsAg negative patients undergoing liver transplantation: clinical significance. Liver Transpl 2004;10:356-62.

[37] Abdelmalek MF, Pasha TM, Zein NN, Persing DH, Wiesner RH, Douglas DD. Subclinical reactivation of hepatitis B virus in liver transplant recipients with past exposure. Liver Transpl 2003;9:1253-7.

[38] Vento S, Cainelli F, Longhi MS. Reactivation of replication of hepatitis $\mathrm{B}$ and $\mathrm{C}$ viruses after immunosuppressive therapy: an unresolved issue. Lancet Oncol 2002;3:333-40.

[39] Esteve M, Saro C, Gonzalez-Huix F, Suarez F, Forne M, Viver JM. Chronic hepatitis B reactivation following infliximab therapy in Crohn's disease patients: need for primary prophylaxis. Gut 2004;53:1363-5.

[40] Zanati SA, Locarnini SA, Dowling JP, Angus PW, Dudley FJ, Roberts SK. Hepatic failure due to fibrosing cholestatic hepatitis in a patient with pre surface mutant hepatitis B virus and mixed connective tissue disease treated with prednisolone and chloroquine. J Clin Vir 2004;31:53-7.

[41] Calabrese LH, Zein NN, Vassilopoulos D. Hepatitis B reactivation with immunosuppressive therapy in rheumatic disease: assessment and preventive strategies. Ann Rheum Dis 2006;65:983-9.

[42] Buttgereit F, da Silva JA, Boers M, Burmester GR, Cutolo M, Jacobs J, et al. Standardised nomenclature for glucocorticoid dosages and glucocorticoid treatment regimens: current questions and tentative answers in rheumatology. Ann Rheum Dis 2002;61:718-22.

[43] Alberti A, Clumeck N, Collins S, Gerlich W, Lundgren J, Palù G, et al. Short statement of the first European Consensus Conference on the treatment of chronic hepatitis B and C in HIV co-infected patients. J Hepatol 2005;42:615-24.

[44] Puoti M, Torti C, Bruno R, Filice G, Carosi G. Natural history of chronic hepatitis B in co-infected patients. J Hepatol 2006;44:S65-70. 